\title{
116.
}

\section{CORRECTION OF TWO THEOREMS RELATING TO THE PORISM OF THE IN-AND-CIRCUMSCRIBED POLYGON.}

[From the Philosophical Magazine, vol. vI. (1853), pp. 376-377.]

THE two theorems in my "Note on the Porism of the in-and-circumscribed Polygon" (see August Number), [115], are erroneous, the mistake arising from my having inadvertently assumed a wrong formulæ for the addition of elliptic integrals. The first of the two theorems (which, in fact, includes the other as a particular case) should be as follows :-

Theorem. The condition that there may be inscribed in the conic $U=0$ an infinity of $n$-gons circumscribed about the conic $V=0$, depends upon the development in ascending powers of $\xi$ of the square root of the discriminant of $\xi U+V$; viz. it this square root be

$$
A+B \xi+C \xi^{2}+D \xi^{3}+E \xi^{4}+F \xi^{5}+G \xi^{6}+H \xi^{7}+\ldots,
$$

then for $n=3,5,7$, \&c. respectively, the conditions are

$$
|C|=0, \quad\left|\begin{array}{ll}
C, & D \\
D, & E
\end{array}\right|=0, \quad\left|\begin{array}{ccc}
C, & D, & E \\
D, & E, & F \\
E, & F, & G
\end{array}\right|=0, \& c \text {; }
$$

and for $n=4,6,8$, \&c. respectively, the conditions are

$$
|D|=0, \quad\left|\begin{array}{ll}
D, & E \\
E, & F
\end{array}\right|=0, \quad\left|\begin{array}{ccc}
D, & E, & F \\
D, & F, & G \\
F, & G, & H
\end{array}\right|=0, \& c .
$$

The examples require no correction; since for the triangle and the quadrilateral respectively, the conditions are (as in the erroneous theorem) $C=0, D=0$. 
The second theorem gives the condition in the case where the conics are replaced by the circles $x^{2}+y^{2}-R^{2}=0$ and $(x-a)^{2}+y^{2}-r^{2}=0$, the discriminant being in this case

$$
-(1+\xi)\left\{r^{2}+\xi\left(r^{2}+R^{2}-a^{2}\right)+\xi^{2} R^{2}\right\} .
$$

As a very simple example, suppose that the circles are concentric, or assume $u=0$; the square root of the discriminant is here

$$
(1+\xi) \sqrt{r^{2}+\overline{R^{2} \xi}}
$$

and putting for shortness $\frac{R^{2}}{r^{2}}=\alpha$, we may write

$$
A+B \xi+\ldots=(1+\xi) \sqrt{1+\alpha \xi},
$$

that is, $A=1, \quad B=\frac{1}{2} \alpha+1, \quad C=-\frac{1}{8} \alpha^{2}+\frac{1}{2} \alpha^{2}, \quad D=\frac{1}{16} \alpha^{3}-\frac{1}{8} \alpha^{2}, \quad E=-\frac{5}{128} \alpha^{4}+\frac{1}{16} \alpha^{3}, \quad \&$ c. ; thus in the case of the pentagon,

$$
\begin{aligned}
C E-D^{2} & =\frac{1}{1024} \alpha^{4}\left\{(\alpha-4)(5 \alpha-8)-4(\alpha-2)^{2}\right\} \\
& =\frac{1}{1024} \alpha^{4}\left(\alpha^{2}-12 \alpha+16\right),
\end{aligned}
$$

and the required condition therefore is

$$
\alpha^{2}-12 \alpha+16=
$$

It is clear that, in the case in question,

$$
\frac{r}{R}=\cos 36^{\circ}=\frac{1}{4}(\sqrt{5}+1)
$$

that is,

$$
\frac{R}{r}=\sqrt{5}-1, \text { or }(R+r)^{2}-5 r^{2}=0,
$$

viz.

$$
(\sqrt{\alpha}+1)^{2}-5=0, \text { or } \alpha+2 \sqrt{\alpha}-4=0,
$$

the rational form of which is

$$
\alpha^{2}-12 \alpha+16=0 \text {, }
$$

and we have thus a verification of the theorem for this particular case.

2 Stone Buildings, Oct. 10, 1853. 